\title{
An angiogenic role for the human peptide antibiotic LL-37/hCAP-18
}

\author{
Rembert Koczulla, ${ }^{1,2}$ Georges von Degenfeld, ${ }^{2}$ Christian Kupatt, ${ }^{2}$ Florian Krötz, ${ }^{3}$ \\ Stefan Zahler, ${ }^{3}$ Torsten Gloe, ${ }^{3}$ Katja Issbrücker, ${ }^{4}$ Pia Unterberger, ${ }^{5}$ Mohamed Zaiou, ${ }^{6,8}$ \\ Corinna Lebherz, ${ }^{2}$ Alexander Karl,, ${ }^{2}$ Philip Raake, ${ }^{2}$ Achim Pfosser, ${ }^{2}$ Peter Boekstegers, ${ }^{2}$ \\ Ulrich Welsch, ${ }^{5}$ Pieter S. Hiemstra, ${ }^{7}$ Claus Vogelmeier, ${ }^{1}$ Richard L. Gallo, ${ }^{6,8}$ \\ Matthias Clauss, ${ }^{4}$ and Robert Bals ${ }^{1,2}$
}

\author{
${ }^{1}$ Hospital of the University of Marburg, Department of Internal Medicine, Division of Pulmonology, \\ Philipps Universtät Marburg, Marburg, Germany \\ ${ }^{2}$ Hospital of the University of Munich-Grosshadern, Medizinische Klinik und Poliklinik I, and \\ ${ }^{3}$ Institute for Physiology, Ludwig-Maximilians Universität, Munich, Germany \\ ${ }^{4}$ Max Planck Institute for Physiological and Clinical Research, Bad Nauheim, Germany \\ ${ }^{5}$ Anatomische Anstalt, Ludwig-Maximilians Universität, Munich, Germany \\ ${ }^{6}$ Veterans Affairs San Diego Healthcare System, San Diego, California, USA \\ ${ }^{7}$ Department of Pulmonology, Leiden University Medical Center, Leiden, The Netherlands \\ ${ }^{8}$ Division of Dermatology, Univerity of California, San Diego, San Diego, California, USA
}

\begin{abstract}
Antimicrobial peptides are effector molecules of the innate immune system and contribute to host defense and regulation of inflammation. The human cathelicidin antimicrobial peptide LL-37/hCAP-18 is expressed in leukocytes and epithelial cells and secreted into wound and airway surface fluid. Here we show that LL-37 induces angiogenesis mediated by formyl peptide receptor-like 1 expressed on endothelial cells. Application of LL-37 resulted in neovascularization in the chorioallantoic membrane assay and in a rabbit model of hind-limb ischemia. The peptide directly activates endothelial cells, resulting in increased proliferation and formation of vessel-like structures in cultivated endothelial cells. Decreased vascularization during wound repair in mice deficient for CRAMP, the murine homologue of LL-37/hCAP-18, shows that cathelicidin-mediated angiogenesis is important for cutaneous wound neovascularization in vivo. Taken together, these findings demonstrate that LL-37/hCAP-18 is a multifunctional antimicrobial peptide with a central role in innate immunity by linking host defense and inflammation with angiogenesis and arteriogenesis.
\end{abstract}

J. Clin. Invest. 111:1665-1672 (2003). doi:10.1172/JCI200317545.

\section{Introduction}

Antimicrobial peptides are effector molecules of the innate immune system with microbicidal and proinflammatory activities $(1,2)$. Cathelicidins are one family of antimicrobial peptides characterized by conserved propeptide sequences that were identified in several mammalian species. LL-37/hCAP-18 is the only antimicrobial peptide of the cathelicidin family iden-

Received for publication December 4, 2002, and accepted in revised form March 19, 2003.

Address correspondence to: Robert Bals, Department of Internal Medicine, Division of Pulmonology, Hospital of the University of Marburg, Baldingerstrasse 1, 35043 Marburg, Germany. Phone: 49-0-6421-2866451; Fax: 49-0-6421-2868987;

E-mail: bals@mailer.uni-marburg.de.

Conflict of interest: The authors have declared that no conflict of interest exists.

Nonstandard abbreviations used: cathelin-related antimicrobial peptide (CRAMP); formyl peptide receptor-like 1 (FPRL1); phospholipase C (PLC); human umbilical vein endothelial cell (HUVEC); human neutrophil peptide-1 (HNP-1); platelet endothelial cell adhesion molecule-1 (PECAM-1);

chorioallantoic membrane (CAM); $N$-formyl-methionylleucyl-phenylalanine (fMLP); extracellular signal-regulated kinase-1 (Erk-1). tified from humans (3). It is expressed in leukocytes such as neutrophils, monocytes, NK cells, $\gamma \delta$ T cells, and B cells (4-8), and in epithelial cells of the testis, skin $(8,9)$, the gastrointestinal tract, and the respiratory tract (4). The 37-amino acid-long $C$ terminus represents the antimicrobially active peptide and is referred to as LL-37. LL-37 has been identified in plasma (10), in the airway surface fluid $(4,11)$, and in wound secretions (9). The peptide is induced by inflammatory or infectious stimuli and displays direct antimicrobial activity by interaction with the cell membranes of microorganisms (2). Mice with disrupted Cnlp, the gene coding for CRAMP (cathelinrelated antimicrobial peptide), showed increased susceptibility to skin infections with group A Streptococcus (12). CRAMP shows several close similarities to human LL-37, including an $\alpha$-helical conformation, comparable spectra of antimicrobial activity, and analogous tissue distribution. A deficiency of LL-37/hCAP-18 in the skin of patients with atopic dermatitis may account for the increased susceptibility to skin infections (13). Recently, LL-37 was found to bind to formyl peptide receptor-like 1 (FPRL1), a G protein-coupled, seventransmembrane cell receptor found on macrophages, 
neutrophils, and subsets of lymphocytes (14). Cathelicidin antimicrobial peptides seem to play a central role in host defense by direct antimicrobial activity and modulation of inflammation. The role of antimicrobial peptides as effector molecules of innate immunity is widening from solely endogenous antibiotics to multifunctional mediators that provide a first line of host defense, modify the local inflammatory response, and activate adaptive immunity.

The formation of new blood vessels is a prerequisite of tissue repair and wound healing $(15,16)$. Vascularization is initiated by various factors ranging from mechanical stress and hypoxia to the presence of soluble inflammatory mediators (16) and involves the sprouting of small capillaries (angiogenesis) and the growth of preexisting vessels (arteriogenesis). Here we demonstrate that LL-37 induces functionally important angiogenesis and arteriogenesis. Furthermore, we provide evidence that the angiogenic activity of LL-37 is mediated by a direct action on endothelial cells.

\section{Methods}

Cell proliferation assays and Matrigel assays. Human umbilical vein endothelial cells (HUVECs; 104) (17) seeded on six-well plastic dishes were stimulated with the following substances in different concentrations: LL-37 (37 C-terminal amino acids), WKYMVm (W peptide) (500 $\mathrm{ng} / \mathrm{ml}$; Humboldt University, Berlin, Germany), VEGF $_{165}(5 \mathrm{ng} / \mathrm{ml}$; Sigma-Aldrich Chemie $\mathrm{GmbH}$, Munich, Germany), a scrambled version of LL-37 called sLL-37 (RSLEGTDRFPFVRLKNSRKLEFKDIKGIKREQFVKIL), hBD-3 synthesized as described earlier (18), human $\alpha$-defensins isolated from neutrophil granules as a mixture of human neutrophil peptide-1 (HNP-1), -2 , and -3 , as described previously (19), and the recombinant hCAP-18 (20). After 72 hours, the cells were detached and counted. For inhibition experiments we applied human serum (10\%), pertussis toxin $(10 \mathrm{ng} / \mathrm{ml}$, Sigma-Aldrich Chemie $\mathrm{GmbH}$ ), an antiserum against FPRL1 (21) or VEGF (Sigma-Aldrich Chemie GmbH), or inhibitors of MAPK extracellular signal-regulated kinase (PD98059; $10 \mu \mathrm{M}$ ), p38 MAPK (SB203580; 10 $\mu \mathrm{M})$, phospholipase C (PLC) (U-7312; $10 \mu \mathrm{M})$, PI3K (wortmannin; $100 \mathrm{nM}$ ), and PKC (GF109203X; $0.3 \mu \mathrm{M}$ ). HUVECs were cultivated on Matrigel support (Becton Dickinson and Co., Franklin Lakes, New Jersey, USA) in serum-free medium. After 18 hours, the cultures were inspected for the formation of rings and cords. VEGF levels in the medium were determined by ELISA.

Hamster aortic ring sprouting. Aortae from golden Syrian hamsters were cross-sectioned into rings and mounted onto plastic dishes. Matrigel (150 $\mu \mathrm{l}$; BD Biosciences, Bedford, Massachusetts, USA) was placed on top and allowed to gel. After 6 days, the aortic rings were fixed with $3.7 \%$ formaldehyde and analyzed under an inverted microscope.

Measurement of intracellular calcium. HUVECs grown on glass slides were loaded with $2 \mu \mathrm{M}$ Fura-2 AM (Molecular Probes Inc., Eugene, Oregon, USA). After the wash- es, the cells were observed on a fluorescence microscope (Axiovert 100; Carl Zeiss GmbH, Oberkochen, Germany) by alternative illumination at $340 \mathrm{~nm}$ and 380 $\mathrm{nm}$ by a monochromator (Till Photonics GmbH, Munich, Germany). The ratios of the fluorescence intensities were calculated representing the relative intracellular calcium concentration. The test substances were locally applied into the buffer.

$N F-\kappa B$ translocation assay. HUVECs were stimulated with peptides or TNF- $\alpha$ as positive control for 4 hours. Activation of NF- $\kappa \mathrm{B}$ was determined by the distribution of its subunit $\mathrm{p} 65$ between the cytoplasm and nucleus in immunofluorescence images as described previously (17).

Apoptosis of endothelial cells. Apoptosis of HUVECs was measured by quantifying annexin $\mathrm{V}$ binding by flow cytometry. HUVECs were incubated with the respective peptide $(5 \mu \mathrm{g} / \mathrm{ml})$ for 5 hours and then carefully detached from the culture dish using citrate buffer. The following staining procedure was performed according to the manufacturer's handbook (Apoptosis Detection Kit I; PharMingen, Heidelberg, Germany).

Wound model in Cnlp-deficient mice. Age- and sexmatched 129/SVJ mice with disrupted genes for Cnlp (12) and wild-type mice were sacrificed 3 days after a 1 -cm-long linear skin incision was made, as previously described (22). Frozen sections were immunolabeled using rat mAb's against murine platelet endothelial cell adhesion molecule-1 (PECAM-1) (clone MEC 13.3; PharMingen, San Diego, California, USA). Blood vessels were counted using a secondary biotinylated goat anti-rat IgG (Vectastain ABC Elite; Vector Laboratories, Burlingame, California, USA).

Chorioallantoic membrane assay. Chorioallantoic membrane (CAM) assays were performed in hen eggs as described previously (23). Dried aliquots of a mixture of $5 \mu \mathrm{l}$ solvent (water), with or without peptide, with 5 $\mu \mathrm{l}$ of $1 \%$ methylcellulose were placed onto the CAMs and incubated for 3 days. After injection of 20\% Luconyl black 0600 (BASF, Ludwigshafen, Germany), photographs were taken. CAMs were fixed, embedded in paraffin, sectioned, stained with a Masson-Goldner solution, and vessels were counted in hot spots as described previously (24).

Rabbit model of angiogenesis. German/Giant rabbits $(6.5 \pm 0.4 \mathrm{~kg})$ were subjected to excision of the proximal external iliac, the popliteal, and the distal saphenous arteries (25). After 7 days, $200 \mu \mathrm{g}$ LL-37, $200 \mu \mathrm{g}$ sLL-37, $50 \mu \mathrm{g}$ bFGF/20 $\mu \mathrm{g}$ VEGF, or PBS alone were injected in five portions into the muscles of the medial thigh and the calf (five injection sites; $n=4$ /group). Seven and 35 days after surgery, angiography was performed by injection of contrast agent into the abdominal aorta (4 $\mathrm{ml}$ at $2 \mathrm{ml} / \mathrm{s}$; cineangiography 25 frames/s) (Siemens, Munich, Germany). Collateral growth was defined as the increase in angiographic collateral score between 7 and 35 days after initial surgery (assessed by counting the contrast-opacified collateral vessels in the medial thigh using a grid overlay) (26). Blood flow 
was assessed by cinedensitometry of the passage time of the contrast agent between the common iliac artery and the anterior tibial artery (27). Tissue specimens were sampled from the gastrocnemius and fibularis muscles, capillaries were detected by alkaline phosphatase staining with indoxyl-tetrazolium, and capillary density was assessed as ratio of capillaries to muscle fibers. Tissue sections were immunostained for the presence of cells positive for CD5, CD11b, CD68, or myeloperoxidase (Serotec $\mathrm{GmbH}$, Dusseldorf, Germany). Numbers of positive cells were counted in ten microscopic fields per section.

Expression of FPRL1. RT-PCR was performed using total RNA from HUVECs, macrophages, and BEAS-2B cells, using Trizol reagent and the Superscript II reverse transcriptase system (both from Life Technologies Inc., Karlsruhe, Germany) and the following primers based on the GenBank file (NM001462): sense, 5'-GAC CTT GGA TTC TTG CTC TAG TC- $3^{\prime}$ and antisense, $5^{\prime}$-CCA TCC TCA CAA TGC CTG TAA C- $3^{\prime}$, as described earlier (28). For immunohistochemistry, formalin-fixed and paraffinembedded human lung tissue was sectioned and incubated with rabbit antiserum to FPRL1 or control serum. Bound Ab's were visualized using a peroxidase-labeled secondary Ab and AEC Single Solution (Zymed, Berlin, Germany). Western blots were prepared for analysis as described previously using 10\% PAGE gels (4).

Statistical analysis. Values are displayed as mean plus or minus SEM. Comparisons between groups were analyzed by the $t$ test (two-sided) or ANOVA for experiments with more than two subgroups. Post hoc range tests were performed with the $t$ test (two-sided) with a Bonferroni adjustment. Results were considered statistically significant for $P$ values less than 0.05 .

\section{Results}

LL-37 stimulates physiologic and pathologic angiogenesis in vivo. To test the hypothesis that LL-37 may play a role in induction of angiogenesis, we analyzed activities of LL-37 in different in vivo models for angiogenesis and arteriogenesis. When the peptide was applied in the CAM assay, a model for physiologic angiogenesis, a significant induction of vessel growth was observed at doses of $5 \mu \mathrm{g} /$ pellet (Figure 1, a-c). LL-37 induced vessel growth inside CAM areas beneath the pellet, which revealed a typical wheel spoke-like structure similar to the positive control bFGF (Figure 1a) (29). A quantitative analysis revealed a large increase of erythrocytefilled blood vessels and connective tissue in LL-37-treated samples (Figure 1b). A scrambled version of LL-37, called sLL-37, resulted in no increase of blood vessels in comparison with the solvent control (Figure 1, a-c).

In addition, we applied LL-37 in a rabbit hind-limb model to assess the effect of the peptide on both angiogenesis and the induction of collateral circulation under pathologic conditions. In this model angiogenesis and collateral vessel growth (arteriogenesis) are induced by excision of the femoral artery. Seven days after surgical excision of the femoral artery, baseline measurements were performed, and LL-37, control peptides, or buffer were injected into the ischemic hind limb. Four weeks after injection we found significantly increased collateral vessel growth in the ischemic hind limbs of the LL-37 group as compared with animals injected with sLL-37 or buffer alone (Figure 2, a-e). Quantitative histologic evaluation of the muscles of the limb revealed higher capillary density in the calf muscles of the animals treated with the antimicrobial peptide, indicating that not only arteriogenesis but also angiogenesis is induced in this model (Figure 2f). Finally, we found increased blood flow velocity between the internal iliac artery and the anterior tibial artery in the LL-37-exposed animals as compared with the negative control groups (Figure $2 \mathrm{~g}$ ). Immunohistologic analysis a

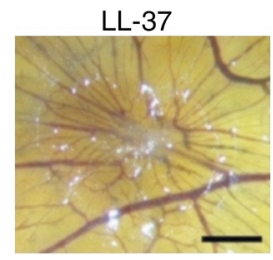

Solvent
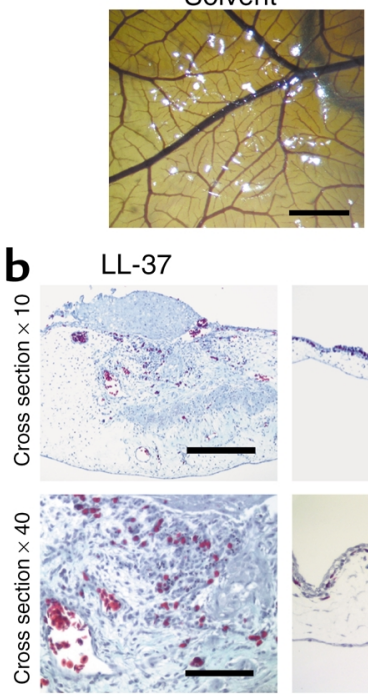

C

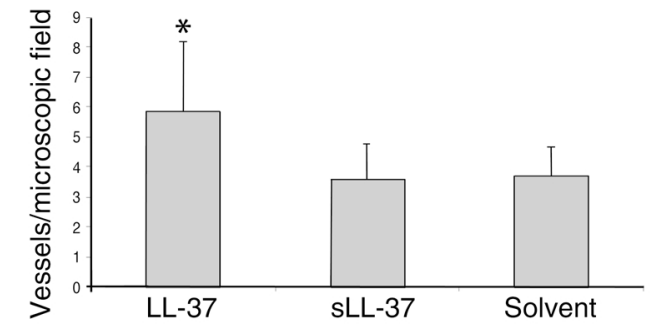

\section{Figure 1}

LL-37 induces physiologic angiogenesis in the CAM assay. (a) LL-37 ( $5 \mu \mathrm{g} /$ pellet) induces the formation of wheel spoke-like vessel formation in comparison with the scrambled control peptide SLL-37 or the solvent. bFGF was used as positive control. Bars: $1 \mathrm{~mm}$. (b) Cross sections of CAMs. LL-37 increases the number of erythrocyte-filled vessels (Masson-Goldner stain of erythrocyte-filled vessels). Bars: $250 \mu \mathrm{m}$ or $62 \mu \mathrm{m}$ in the $\times 10$ and $\times 40$ micrographs, respectively. (c) The numbers of erythrocyte-filled vessels were counted in hot spots. ${ }^{*} P<0.05$ as compared with the solvent group $(n=6 /$ group; three sections per CAM). 


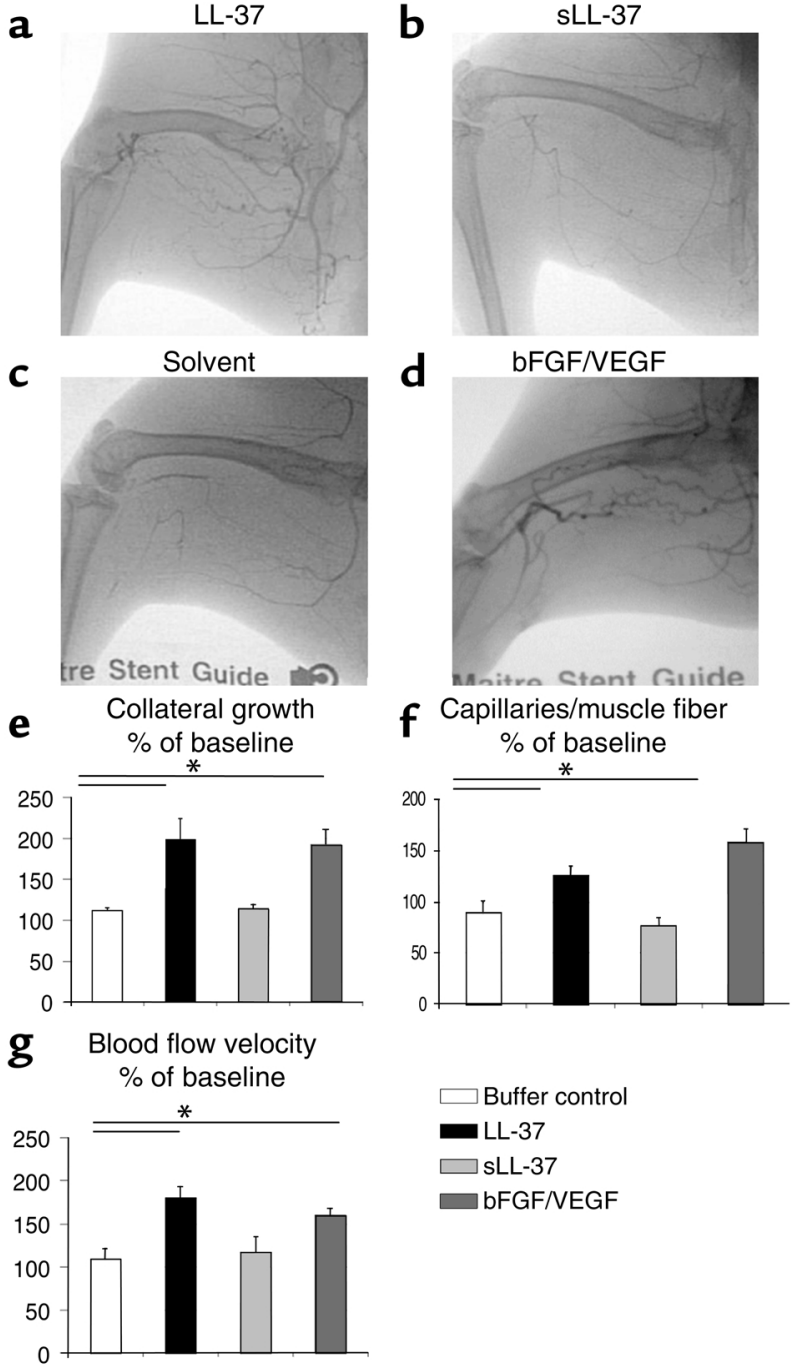

of inflammatory cells 3 days or 4 weeks after injection revealed no significant increase of CD5, CD11b, CD68, or myeloperoxidase-positive cells (data not shown). These data show that LL-37/hCAP-18 induces angiogenesis in vivo in both models of physiologic and pathologic angiogenesis and that this is accompanied by functionally relevant increased blood supply.

Murine CRAMP is necessary for normal wound vascularization. The cathelicidin antimicrobial peptide CRAMP is the putative murine homologue of LL-37/hCAP- 18 . To test whether mice with disrupted Cnlp genes coding for CRAMP have impaired vascularization of wounds, we injured animals by full-thickness aseptic linear incisions. As described previously, this method of injury results in a large increase in cathelicidins at the wound edge in both normal mice and humans (22). Neovascularization at the wound edge was observed histologically 3 days after injury. CD31 (PECAM-1) expression on endothelial cells was evaluated by immunohistochemistry to clearly identify vascular structures. We found that CRAMP-deficient mice had significantly decreased vascular structures at the wound edge when compared with normal animals matched for age and sex (Figure 3,

\section{Figure 2}

LL-37 induces angiogenesis and arteriogenesis in the rabbit hind-limb model. (a-d) Application of the peptide in a rabbit hind-limb model resulted in increased collateral growth in the LL-37-treated animals (a) as compared with the sLL-37-treated animals (b) or the buffer control group (c). bFGF/VEGF was used as positive control (d). (e) Collateral growth was significantly increased in the LL-37 group. (f) Tissue specimens from the calf (gastrocnemius muscle) revealed higher capillary density in the treated group as compared with the control group. (g) Blood flow velocity as assessed by cinedensitometry of the passage time of the contrast agent between the internal iliac artery and the anterior tibial artery was significantly augmented in the LL-37 group. ${ }^{*} P<0.05$ as compared with the buffer control ( $n=4 /$ group).

$a-c)$. No apparent difference was seen in the total number of vessels observed in the normal dermis distant from the site of injury. These results provide strong evidence that cathelicidins are necessary for wound vascularization in vivo.

LL-37 induces angiogenesis by a direct effect on endothelial cells. To address the question whether the angiogenic activities of LL-37 can be explained by a direct action on endothelial cells we applied LL-37 in several in vitro and ex vivo models of angiogenesis. We measured the effect of LL-37 on proliferation of HUVECs and found that addition of the peptide resulted in a dose-dependent increase of cell proliferation with a minimal effective concentration as low as $50 \mathrm{ng} / \mathrm{ml}$ (Figure 4a). The proliferative function of LL-37 could not be blocked by the presence of $10 \%$ human serum (Figure $4 a$ ), which was demonstrated previously to abrogate the antimicrobial activity of LL-37 (30). At equimolar concentrations, other peptides, including HNPs, hBD-3, and the propeptide hCAP-18, had no effect on endothelial proliferation
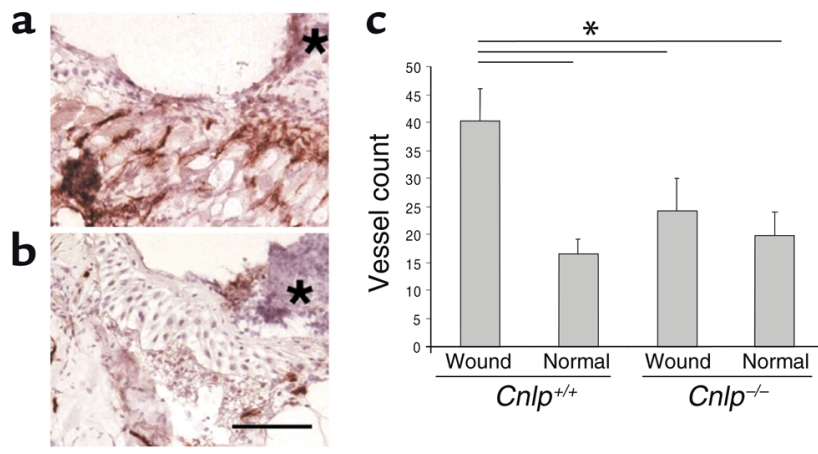

\section{Figure 3}

Mice with disrupted Cnlp gene show decreased wound revascularization. ( $\mathbf{a}$ and $\mathbf{b}$ ) Vascular structures are identified in microsections by CD31 immunostaining at the wound edge 3 days after full-thickness aseptic injury. An asterisk denotes the wound site and location of crust. Wild-type 129/SvJ mice show multiple vascular structures in granulation tissue at the margin of the repairing wound (a). Mice with homozygous deletion of Cnlp, therefore lacking CRAMP, have fewer vessels at an identical location relative to the wound (b). The bar $=12.5 \mu \mathrm{m}$ in both micrographs. (c) Numbers of vessels in the skin near the site of injury are significantly decreased in $\mathrm{Cn} / \mathrm{p}^{-1-} \mathrm{com}$ pared with wild-type mice, but are similar distal to the wound in each group ( ${ }^{*} P<0.05 ; n=3$ /group, three sections per animal). 


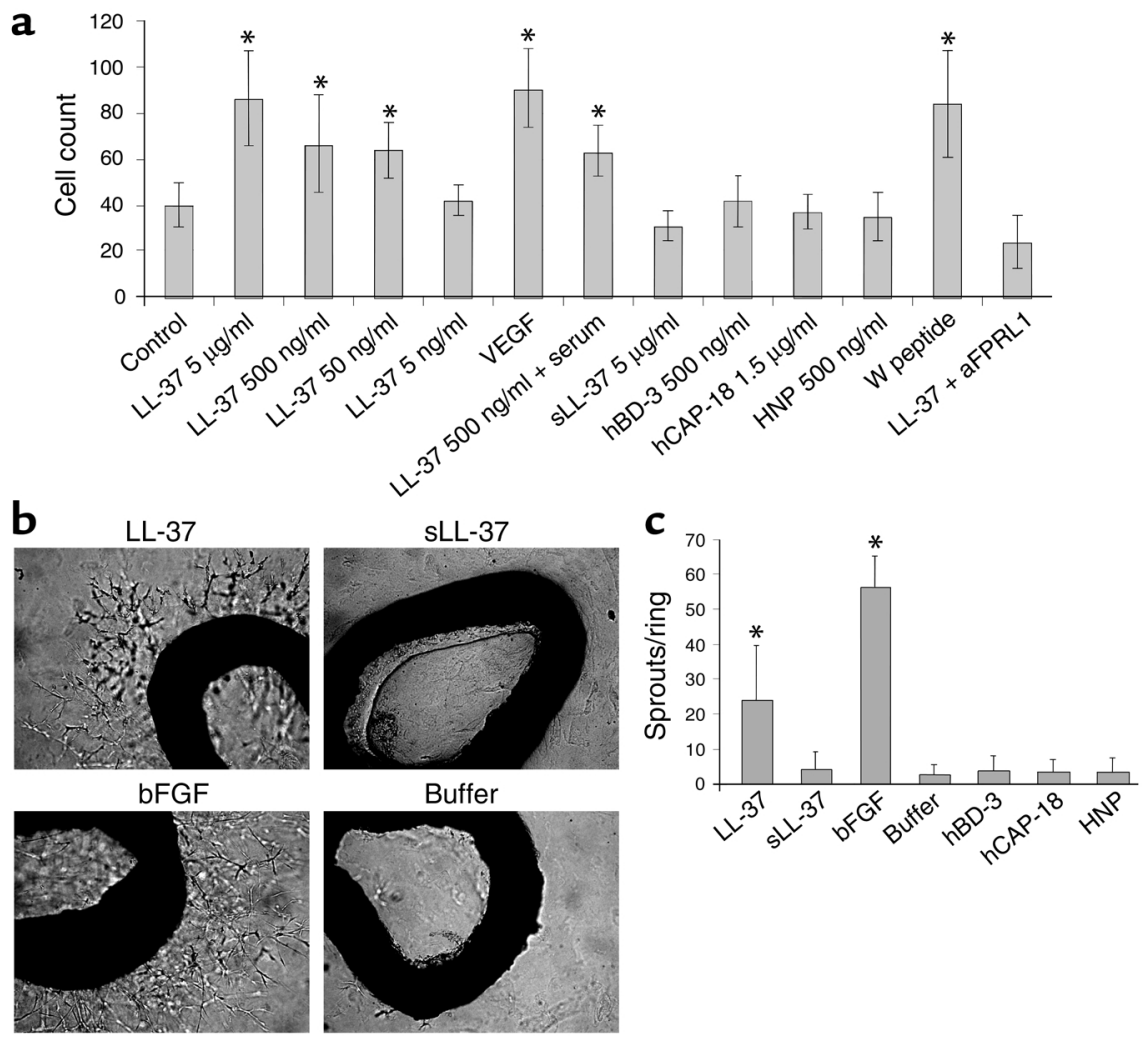

\section{Figure 4}

Effects of LL-37 on endothelial cells in vitro. (a) In vitro proliferation assays. Different concentrations of LL-37 and controls were added to cultivated HUVECs. Numbers at the left of the graph indicate the numbers of cells per microliter of volume after 72 hours. VEGF was used as positive control. Additionally, we tested sLL-37, HNP-1, -2, and -3, hBD-3, and the propeptides hCAP-18. In vitro proliferation was not inhibited by human serum. Application of the FPRL1 agonist peptide WKYMVm (W peptide) results in increased growth of cell numbers. Addition of antiserum to FPRL1 blunted increased cellular proliferation induced by LL-37. ${ }^{*} P<0.05$ as compared with the control group ( $n=10 /$ group). (b and $\mathbf{c}$ ) Hamster aortic ring-sprouting assay. The addition of LL-37 to the culture medium resulted in increased sprouting from the aortic rings as compared with control groups that received bFGF or no peptide (b). ${ }^{*} P<0.05$ in the LL-37 and bFGF groups as compared with the controls ( $n=7 /$ group) (c). aFPRL1, antiserum plus FPRL1.

(Figure 4a). To investigate whether LL-37 might influence angiogenesis by induction of VEGF synthesis, we determined VEGF levels in the cell culture supernatants by ELISA and found no increased concentrations after stimulation with LL-37 (data not shown). Furthermore, coincubation with neutralizing anti-VEGF Ab's did not reduce the LL-37-induced proliferation of endothelial cells (data not shown). Next, we measured the formation of vascular structures by applying LL-37 in an in vitro Matrigel assay and observed the formation of visible rings and cords of endothelial cells in response to LL-37 but not to the control substances, including other antimicrobial peptides (data not shown). In addition, LL-37 stimulated outgrowth of endothelial cells from hamster aortic rings in an ex vivo sprouting assay similar to bFGF (Figure 4, b and c). HNPs or hCAP-18 had no effect on the sprouting of endothelial cells (Figure 4c). These data demonstrate that LL-37/hCAP-18 directly activates endothelial cells and induces endothelial sprout formations both in vitro and ex vivo.

Angiogenic activities of LL-37 in endothelial cells are mediated by FPRL1 signaling. We next analyzed the mechanisms involved in the angiogenic activity of LL-37. Because the peptide LL-37 was shown to bind to FPRL1 (14), we tested whether HUVECs express FPRL1. Indeed, we observed expression of FPRL1 in HUVECs as a 620 -bp product of a RT-PCR (Figure 5a) and a positive signal in a Western blot using antiserum against FRPL1 (Figure 5b). DNA sequence analysis of the PCR product confirmed $100 \%$ identity with the corresponding cDNA sequence of FPRL1 (data not shown). In the next step we analyzed the tissue distribution of FPRL1 in the human lung, an organ rich in vessels of different types and a variety of other cell types, such as epithelial, inflammatory, and immune cells. We observed FPRL1 not only in macrophages, neutrophils, and airway epithelium, but also in endothelial cells (Figure 5c). Sections stained with a control serum did not show any positive staining (Figure $5 \mathrm{~d}$ ).
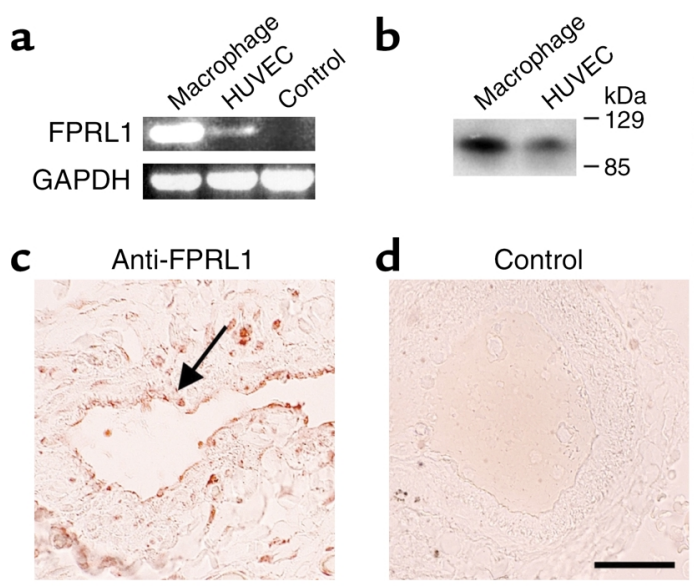

Figure 5

Endothelial cells express FPRL1 in vitro and in vivo. (a) Detection of FPRL1 transcripts in cultivated HUVECs by RT-PCR. Control is the BEAS-2B cell line. (b) Detection of FPRL1 protein in HUVECs by Western blot analysis using a specific antiserum. (c) Immunohistochemistry revealed expression in endothelial cells (arrow) of sections of lung tissue. (d) Control serum revealed no positive staining. Bar: $60 \mu \mathrm{m}$. 

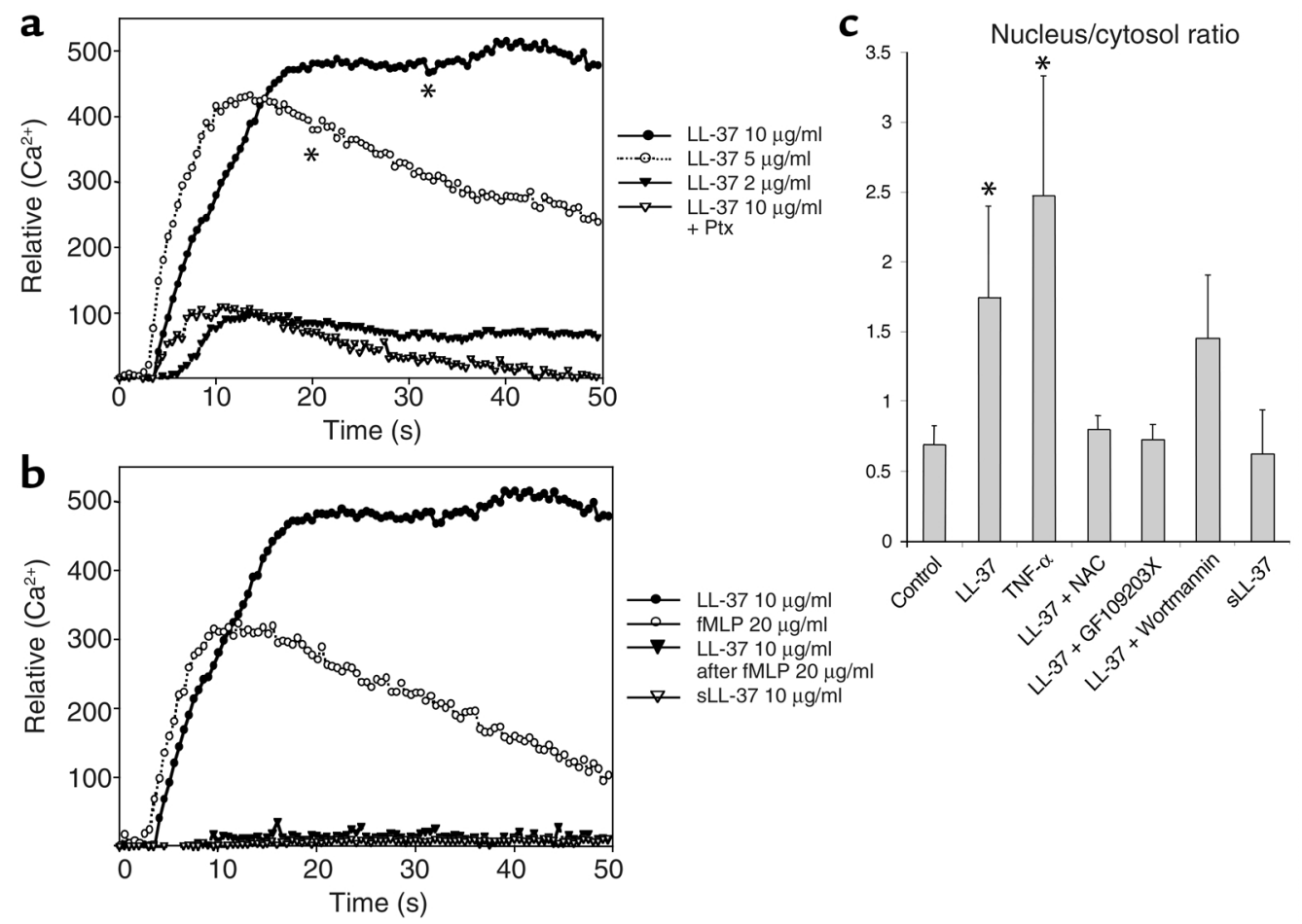

Figure 6

LL-37 binds to endothelial FPRL1 and induces cellular signaling. ( $\mathbf{a}$ and $\mathbf{b}$ ) LL-37 induces Ca ${ }^{2+}$ flux in HUVECs. Fura-2-loaded HUVECs were stimulated with LL-37, and relative levels of intracellular $\mathrm{Ca}^{2+}$ were monitored using a calcium-imaging system. Local application of LL-37 led to a concentration-dependent increase of intracellular calcium. Preincubation of the cells with pertussis toxin (Ptx) resulted in partial inhibition of the $\mathrm{Ca}^{2+}$ flux (a). Preincubation with $\mathrm{fMLP}$ cross-desensitized the LL-37-induced Ca ${ }^{2+}$ mobilization; sLL-37 had no effect (b). (c) LL-37 activates NF-KB in endothelial cells. This response can be inhibited by GF109203X and the addition of $N$-acetylcystein. ${ }^{*} P<0.05$ as compared with the control group $(n=8)$. NAC, $N$-acetylcystein.

To provide further evidence for a functional role of FPRL1 in LL-37-induced endothelial activities, the FPRL1-agonistic hexapeptide WKYMVm (31) was applied to HUVECs and was found to stimulate proliferation to an extent similar to LL-37 (Figure 4a). The involvement of FPRL1 in endothelial stimulation by LL-37 was supported by inhibition studies using pertussis toxin, an inhibitor of $\mathrm{G}$ protein-coupled receptors that abolished effects of LL-37 on endothelial cells, including proliferation (data not shown). Intriguingly, a neutralizing antiserum to FPRL1 completely blocked the proliferative effect of LL-37 in vitro, whereas a control serum had no effect (Figure 4a). These results show that FPRL1 is expressed on endothelial cells and is necessary and sufficient to mediate the angiogenic activity of LL-37.

Downstream events of FPRL1 activation involve increased intracellular calcium (32) and diverse signaling pathways, including the activation of the cytosolic phospholipase A2, PI3K, and PLC (33). Using a calcium-imaging system we found that addition of LL-37 to the medium of cultured endothelial cells at final concentrations from 2 to $10 \mu \mathrm{g} / \mathrm{ml}$ resulted in an increase of intracellular $\mathrm{Ca}^{2+}$ (Figure 6a). The LL-37-specific increase of intracellular $\mathrm{Ca}^{2+}$ could be cross-desensitized by preincubation with $\mathrm{N}$-formyl-methionyl- leucyl-phenylalanine (fMLP-peptide), which also induced a rise in intracellular calcium when solely applied to the cells (Figure 6b). Also, preincubation with pertussis toxin inhibited the rise in intracellular $\mathrm{Ca}^{2+}$ after application of LL-37 (Figure 6a). In addition, we could demonstrate that LL-37/hCAP-18 induced nuclear translocation of NF- $\mathrm{KB}$ (Figure 6c). This reaction was inhibited by the PKC-inhibitor (GF109203X) and the addition of the antioxidant $N$-acetylcystein (Figure 6c). Addition of GF109203X completely abolished the LL-37-induced increase in proliferation (data not shown). To investigate involvement of MAPK extracellular signal-regulated kinase- 1 and -2 (Erk-1 and -2) and p38 MAPK, we used PD98059 and SB203580, respectively. PD98059, but not SB203580, inhibited the LL-37-mediated endothelial proliferation (data not shown) indicating that Erk-1 and -2 may also be involved in LL-37-elicited effects. Furthermore, we tested the potential involvement of the PI3K/Akt-kinase pathways by using the PI3K inhibitor wortmannin. Although wortmannin only partially reduced NF- $\mathrm{KB}$ translocation (Figure 6c), it strongly reduced LL-37induced endothelial cell growth (data not shown). Additionally, we tested whether antiapoptotic effects of LL-37 might contribute to the proliferative effect; however, we could not detect any effect in the annexin 
V-binding assay (data not shown). Taken together, our data suggest that LL-37 is able to stimulate several signaling pathways involved in endothelial proliferation.

\section{Discussion}

The main finding of our study is the capability of the human cathelicidin peptide LL-37 to induce functionally important angiogenesis. Several lines of evidence suggest that LL-37-induced neovascularization is mediated by a direct effect on endothelial cells involving the specific receptor FPRL1. Our ex vivo studies using the aortic ring assay confirm that a direct activity of LL-37 on endothelial cells is involved in vivo. Application of LL-37 in vivo resulted in vessel growth in models of physiologic and pathologic angiogenesis. In addition, we demonstrate that mice missing the murine homologue of LL-37/hCAP-18 show decreased wound vascularization.

The mechanism of angiogenic activity of LL-37 is dependent on binding of the peptide to FPRL1, a G protein-coupled receptor recently found to mediate cellular responses to LL-37 (14). We demonstrate that endothelial cells express functional FPLR1 and that effects of LL-37 are specifically mediated by FPRL1. Other cationic antimicrobial peptides and the propeptide hCAP-18 did not stimulate endothelial cells. In contrast, the propeptide of the rabbit cathelicidin CAP-18 shows biological activity by blocking lipopolysaccharide-induced activation of leukocytes (34). The angiogenic mechanism of LL-37 is clearly distinct from the one described for PR-39, a porcine cathelicidin peptide, which appears to affect angiogenesis by inhibiting the ubiquitin-proteasome-dependent degradation of HIF-1 $\alpha$ (35). The possibility of an indirect effect of LL-37 by production of VEGF is unlikely because addition of LL-37 to HUVECs did not lead to release of VEGF in the supernatant and neutralizing anti-VEGF did not reduce LL-37-induced endothelial proliferation. Our analysis of signal transduction pathways involved in LL-37-elicited endothelial activation revealed involvement of the PLC- $\gamma / \mathrm{PKC} / \mathrm{NF}-\kappa \mathrm{B}$, the Erk-1 and -2 MAPK, and the PI3K/Akt pathways. As well as its direct angiogenic effect on endothelial cells, LL-37 may initiate vessel growth by attracting neutrophils and monocytes in vivo. These cells contain significant amounts of angiogenic mediators that are released in response to cellular activation $(5,36)$ and are attracted by LL-37 $(5,14)$.

Our findings extend the spectrum of functions of human antimicrobial peptides, demonstrating a direct role in angiogenesis. LL-37/hCAP-18 expression is increased during inflammation and in wound areas (5, $9,11,14,22)$. Significant concentrations of LL-37 are found in body fluids during inflammatory diseases $(9$, 37). In addition to its antimicrobial activities, LL-37/ hCAP functions in galvanizing innate and adaptive immunity by activation of neutrophils, monocytes, and lymphocyte subsets (14). Here we show that LL-37/ hCAP-18 is also able to induce angiogenesis, a process essential for host defense, wound healing, and tissue repair. In experimental, sterile wounds, keratinocytes upregulate LL-37/hCAP-18 within 24 hours after the incision (22). In addition to epithelial cells, the cathelicidin peptide is secreted from inflammatory cell types such as neutrophils and macrophages that invade wound areas. With several cellular sources, the peptide likely reaches high local concentrations that are in the range of antimicrobial, chemoattractant, and angiogenic activity. The angiogenic effect of LL-37 is not inhibited by the presence of human serum as described for the peptide's antimicrobial activity (30) and therefore is likely active in vivo.

In conclusion, the human endogenous peptide antibiotic LL-37/hCAP-18 induces functionally important angiogenesis. This angiogenic activity is mediated by a direct effect on endothelial cells. Together with its antimicrobial and immunomodulatory functions, LL-37/hCAP-18 links inflammation and host defense with angiogenesis. The peptide, its analogues, and other agonists of FPRL1 may prove to be effective agents for induction of therapeutic angiogenesis. The clinical relevance of such an approach is illustrated by the observation that infection and impaired blood supply contribute to a variety of important human diseases, including foot and leg ulcers associated with diabetes or burn wounds.

\section{Acknowledgments}

We thank Charles N. Serhan for providing FPRL1/ LXA4R Ab, Masamoto Murakami (University of California at San Diego) for assistance in processing of mouse wound tissues, and Daniel J. Weiner (University of Pennsylvania) for helpful discussions. This work was supported by an award to R. Bals (Adolf-Windorfer-Preis of the Mukoviszidose e.V. 1999), the Deutsche Forschungsgemeinschaft SPP 1069 (Angiogenese) to M. Clauss, and NIH grants AI48176, AR4576, and a Veterans Affairs Merit Award to R.L. Gallo.

1. Ganz, T. 1999. Defensins and host defense. Science. 286:420-421.

2. Bals, R. 2000. Epithelial antimicrobial peptides in host defense against infection. Respir. Res. 1:141-150.

3. Zanetti, M., Gennaro, R., and Romeo, D. 1995. Cathelicidins: a novel protein family with a common proregion and a variable C-terminal antimicrobial domain. FEBS Lett. 374:1-5.

4. Bals, R., Wang, X., Zasloff, M., and Wilson, J.M. 1998. The peptide antibiotic LL-37/hCAP-18 is expressed in epithelia of the human lung where it has broad antimicrobial activity at the airway surface. Proc. Natl. Acad. Sci. U. S. A. 95:9541-9546.

5. Agerberth, B., et al. 2000. The human antimicrobial and chemotactic peptides LL-37 and alpha-defensins are expressed by specific lymphocyte and monocyte populations. Blood. 96:3086-3093.

6. Gudmundsson, G.H., et al. 1996. The human gene FALL39 and processing of the cathelin precursor to the antibacterial peptide LL-37 in granulocytes. Eur. J. Biochem. 238:325-332.

7. Cowland, J., Johnsen, A., and Borregaard, N. 1995. hCAP-18, a cathelin/pro-bactenecin-like protein of human neutrophil specific granules. FEBS Lett. 368:173-176.

8. Frohm, M., et al. 1997. The expression of the gene coding for the antibacterial peptide LL-37 is induced in human keratinocytes during inflammatory disorders. J. Biol. Chem. 272:15258-15263.

9. Frohm, M., et al. 1996. Biochemical and antibacterial analysis of human wound and blister fluid. Eur. J. Biochem. 237:86-92.

10. Sorensen, O., Cowland, J., Askaa, J., and Borregaard, N. 1997. An ELISA for hCAP-18, the cathelicidine present in human neutrophils and plasma. FEBS Lett. 206:53-59. 
11. Agerberth, B., et al. 1999. Antibacterial components in bronchoalveolar lavage fluid from healthy individuals and sarcoidosis patients. Am. J. Respir. Crit. Care Med. 160:283-290.

12. Nizet, V., et al. 2001. Innate antimicrobial peptide protects the skin from invasive bacterial infection. Nature. 414:454-457.

13. Ong, P.Y., et al. 2002. Endogenous antimicrobial peptides and skin infections in atopic dermatitis. N. Engl. J. Med. 347:1151-1160.

14. Yang, D., et al. 2000. LL-37, the neutrophil granule- and epithelial cellderived cathelicidin, utilizes formyl peptide receptor-like 1 (FPRL1) as a receptor to chemoattract human peripheral blood neutrophils, monocytes, and T-cells. J. Exp. Med. 192:1069-1074.

15. Buschmann, I., and Schaper, W. 2000. The pathophysiology of the collateral circulation (arteriogenesis). J. Pathol. 190:338-342.

16. Carmeliet, P. 2000. Mechanisms of angiogenesis and arteriogenesis. Nat. Med. 6:389-395.

17. Zahler, S., Kupatt, C., and Becker, B.F. 2000. Endothelial preconditioning by transient oxidative stress reduces inflammatory responses of cultured endothelial cells to TNF-alpha. FASEBJ. 14:555-564.

18. Garcia, J.R., et al. 2001. Identification of a novel, multifunctional betadefensin (human beta-defensin 3 ) with specific antimicrobial activity. Its interaction with plasma membranes of Xenopus oocytes and the induction of macrophage chemoattraction. Cell Tissue Res. 306:257-264.

19. Aarbiou, J., et al. 2002. Human neutrophil defensins induce lung epithelial cell proliferation in vitro. J. Leukoc. Biol. 72:167-174

20. Zaiou, M., Nizet, V., and Gallo, R.L. 2003. Antimicrobial and protease inhibitory functions of the human cathelicidin (hCAP-18/LL-37) prosequence. J. Invest. Dermatol. 120:880-890.

21. Fiore, S., and Serhan, C.N. 1995. Lipoxin A4 receptor activation is distinct from that of the formyl peptide receptor in myeloid cells: inhibition of CD11/18 expression by lipoxin A4-lipoxin A4 receptor interaction. Biochemistry. 34:16678-16686.

22. Dorschner, R.A., et al. 2001. Cutaneous injury induces the release of cathelicidin anti-microbial peptides active against group A Streptococcus. J. Invest. Dermatol. 117:91-97.

23. Drexler, H.C., Risau, W., and Konerding, M.A. 2000. Inhibition of proteasome function induces programmed cell death in proliferating endothelial cells. FASEB J. 14:65-77.
24. Issbrucker, K., et al. 2003. p38 MAP kinase - a molecular switch between VEGF-induced angiogenesis and vascular hyperpermeability. FASEB J. 17:262-264.

25. Takeshita, S., et al. 1994. Therapeutic angiogenesis. A single intraarterial bolus of vascular endothelial growth factor augments revascularization in a rabbit ischemic hind limb model. J. Clin. Invest. 93:662-670.

26. Murohara, T., et al. 1998. Nitric oxide synthase modulates angiogenesis in response to tissue ischemia. J. Clin. Invest. 101:2567-2578.

27. Gibson, C.M., et al. 1996. TIMI frame count: a quantitative method of assessing coronary artery flow. Circulation. 93:879-888.

28. Bals, R., et al. 1998. Human beta-defensin 2 is a salt-sensitive peptide antibiotic expressed in human lung. J. Clin. Invest. 102:874-880.

29. Ribatti, D., et al. 1997. New model for the study of angiogenesis and antiangiogenesis in the chick embryo chorioallantoic membrane: the gelatin sponge/chorioallantoic membrane assay. J. Vasc. Res. 34:455-463.

30. Wang, Y., Agerberth, B., Lothgren, A., Almstedt, A., and Johansson, J. 1998. Apolipoprotein A-I binds and inhibits the human antibacterial/cytotoxic peptide LL-37. J. Biol. Chem. 273:33115-33118.

31. Li, B.Q., et al. 2001. The synthetic peptide WKYMVm attenuates the function of the chemokine receptors CCR5 and CXCR4 through activation of formyl peptide receptor-like 1. Blood. 97:2941-2947.

32. Hu, J.Y., et al. 2001. Synthetic peptide MMK-1 is a highly specific chemotactic agonist for leukocyte FPRL1. J. Lenkoc. Biol. 70:155-161.

33. Baek, S.H., et al. 1999. Trp-Lys-Tyr-Met-Val-Met activates mitogen-activated protein kinase via a PI-3 kinase-mediated pathway independent of PKC. Life Sci. 65:1845-1856.

34. Zarember, K.A., et al. 2002. Host defense functions of proteolytically processed and parent (unprocessed) cathelicidins of rabbit granulocytes. Infect. Immun. 70:569-576.

35. Li, J., et al. 2000. PR39, a peptide regulator of angiogenesis. Nat. Med. 6:49-55.

36. Sorensen, O.E., et al. 2001. Human cathelicidin, hCAP-18, is processed to the antimicrobial peptide LL-37 by extracellular cleavage with proteinase 3. Blood. 97:3951-3959.

37. Bals, R., Weiner, D.J., Meegalla, R.L., Accurso, F., and Wilson, J.M. 2001. Salt-independent abnormality of antimicrobial activity in cystic fibrosis airway surface fluid. Am. J. Respir. Cell. Mol. Biol. 25:21-25. 